\title{
Paired Box Protein Pax-2
}

National Cancer Institute

\section{Source}

National Cancer Institute. Paired Box Protein Pax-2. NCI Thesaurus. Code C73692.

Paired box protein PAX-2 (416aa, $\sim 45 \mathrm{kD})$ is encoded by the human PAX2 gene. This soluble, nuclear protein plays a role in transcription by RNA polymerase II and cellular proliferation. 